\title{
O uso seguro da eletrocirurgia no período intraoperatório: evidências para o
}

\section{cuidado de enfermagem}

\author{
The safe use of electrosurgery in the intraoperative period: evidence for nursing care \\ El uso seguro de la electrocirugía en el período intraoperatorio: evidencia para el cuidado de \\ enfermeira
}

Recebido: 15/12/2020 | Revisado: 22/12/2020 | Aceito: 25/12/2020 | Publicado: 01/01/2021

\author{
Jane Keyla Souza dos Santos Macedo \\ ORCID: https://orcid.org/0000-0002-5037-097X \\ Universidade Federal de Alagoas, Brasil \\ E-mail: jkeyla_souza@hotmail.com \\ Eveline Lucena Vasconcelos \\ ORCID: https://orcid.org/0000-0003-3522-6248 \\ Universidade Federal de Alagoas, Brasil \\ E-mail: evelinelucena@gmail.com
}

\begin{abstract}
Resumo
Objetivo: Este estudo objetivou analisar a produção científica publicada a respeito do cuidado de enfermagem frente ao uso da eletrocirurgia, e identificar as contribuições destas para a qualidade da assistência. Metodologia: Trata-se de uma revisão integrativa, utilizando os estudos publicados nos últimos vinte anos nas bases de dados LILACS e MEDLINE® via PubMed®, nas bibliotecas BVS-Enfermagem e Scielo, e no portal Google Acadêmico. O período de coleta de dados ocorreu de 01/08/2020 a 31/10/2020. Resultados: Foram encontrados 24 estudos que ao analisar a essência dos conteúdos constatou-se que em 62,5\% abordaram o conhecimento da equipe de enfermagem acerca do uso da eletrocirurgia. Em aproximadamente $60 \%$ dos artigos referiram a necessidade de adotar medidas de segurança do paciente para minimizar os potenciais riscos da unidade eletrocirurgia, e apenas $33,3 \%$ das publicações abordaram o papel do enfermeiro perioperatório para favorecer e garantir a prevenção e segurança no uso da eletrocirurgia. Neste sentido, emerge a necessidade de se ter uma enfermagem alinhada às necessidades dos pacientes e com capacidade de prevenir as diversas injúrias que ocorrem, principalmente, nas salas de operação. Conclusão: Os resultados deste estudo sinalizam uma produção científica ainda em progressão, e apontam que a equipe de enfermagem se encontra dentre os profissionais que desempenham um importante e indispensável papel no cuidado sobre a eletrocirurgia no período intraoperatório.
\end{abstract}

Palavras-chave: Eletrocirurgia; Enfermagem; Período intraoperatório; Tecnologia aplicada à assistência à saúde.

\begin{abstract}
Objective: This study aimed to analyze the published scientific production regarding nursing care regarding the use of electrosurgery, and to identify their contributions to the quality of care. Methodology: It is na integrative review, using the studies published in the last twenty years in the LILACS and MEDLINE® databases via PubMed, in the VHL- Nursing and Scielo Libraries, and in the Google Scholar portal. The data collection period occurred from 01/08/2020 to 31/10/2020. Results: Twenty-four studies were found that, when analyzing the essence of the contents, it was found that in $62.5 \%$ they addressed the knowledge of the nursing team about the use of electrosurgery. In approximately $60 \%$ of the articles, they mentioned the need to adopt patient safety measures to minimize the potential risks of the electrosurgery unit, and only $33.3 \%$ of the publications addressed the role of the perioperative nurse to favor and guarantee prevention and safety in the use of electrosurgery. In this sense, the need emerges to have a nursing team aligned with the needs of patients andwith the ability to prevent the various injuries that occur, mainly, in the operating rooms. Conclusion: The results of this study indicate a scientific production still in progress, and point out that the nursing team is among the professionals who play na important and indispensable role in the care of electrosurgery in the intraoperative period.
\end{abstract}

Keywords: Electrosurgery; Nursing; Intraoperative period; Technology applied to health care.

\section{Resumen}

Objetivo: Este estudio tuvo como objetivo analizar la producción científica publicada sobre el cuidado de enfermería sobre el uso de la electrocirugía, e identificar sus contribuciones a la calidad de la atención. Metodología: Está en la revisión integradora, utilizando los estudios publicados en los últimos veinte años en las bases de datos LILACS y MEDLINE® vía PubMed, en las Bibliotecas BVS-Nursing y Scielo, y en el portal Google Scholar. El período de recolección de datos ocurrió desde el 01/08/2020 hasta el 31/10/2020. Resultados: Se encontraron veinticuatro 
estudios que, al analizar la esencia de los contenidos, se encontró que en el 62,5\% abordaban los conocimientos del equipo de enfermería sobre el uso de la electrocirugía. En aproximadamente el $60 \%$ de los artículos mencionaron la necesidad de adoptar medidas de seguridad del paciente para minimizar los riesgos potenciales de la unidad de electrocirugía, y solo el 33,3\% de las publicaciones abordó el papel de la enfermera perioperatoria para favorecer y garantizar la prevención y seguridad en el uso de electrocirugía. En este sentido, surge la necesidad de contar con un equipo de enfermería alineado con las necesidades de los pacientes y capaz de prevenir las diversas lesiones que se producen, especialmente en quirófanos. Conclusión: Los resultados de este estudio indican una producción científica aún en progreso, y señalan que el equipo de enfermería se encuentra entre los profesionales que juegan un papel importante e indispensable en el cuidado de la electrocirugía en el período intraoperatorio.

Palabras clave: Electrocirugía; Enfermería; Período intraoperatorio; Tecnología aplicada al cuidado de la salud.

\section{Introdução}

É notório que ao longo da história os profissionais de saúde sempre estiveram buscando aprimorar a assistência, eliminar os possíveis danos, e garantir qualidade e segurança no cuidado, baseando-se no conhecimento técnico-científico associado às tecnologias de cada período (Oliveira, et al., 2017).

Sendo assim, pode-se evidenciar que por meio da evolução da assistência à saúde, observa-se que desde tempos remotos a segurança do paciente é alvo de discussões pelos pesquisados da saúde, pois apesar dos inúmeros benefícios já conhecidos, verifica-se que cada vez mais são frequentes a ocorrência de erros durante o cuidado prestado nos serviços de saúde (Nascimento \& Draganov, 2015, Duarte, et al., 2015; Souza, Monteiro \& Tanaka, 2020).

Mesmo diante das inúmeras modificações e avanços na área da saúde a segurança do paciente continua sendo uma preocupação mundial. Nesta linha, observa-se que a qualidade do cuidado está diretamente relacionada ao serviço prestado e às intervenções assistenciais realizadas pelos profissionais da saúde (Reis, Martins \& Laguardia, 2013; Oliveira, et al., 2017; Souza, Monteiro \& Tanaka, 2020; Jones, et al., 2017).

À medida que as técnicas cirúrgicas foram se desenvolvendo, em consequência dos avanços científicos, procedimentos mais complexos com acesso aos órgãos foi se tornando cada vez mais possível. Logo, considerou-se a eletrocirurgia como um recurso tecnológico de extrema importância nas intervenções cirúrgicas (Farias, et al., 2019; Borges, 2019).

Diante disso, a eletrocirurgia consiste em uma prática utilizada frequentemente em procedimentos cirúrgicos e seus benefícios (capacidade de realizar cortes precisos e coagulação dos tecidos com uma perda de sangue limitada, e melhor visualização do campo operatório) são amplamente difundidos (Sobecc, 2009; Tomasi, 2013).

Essa técnica baseia-se nos princípios da diatermia em que o tecido corpóreo se situa entre duas placas metálicas e através delas ocorre à passagem das ondas de alta frequência provocando a liberação de cargas elétricas no interior das células expostas (Claudio, et al., 2017; Olímpio, Sousa \& Ponte, 2016; Tramontini, et al., 2016).

Sendo assim, quando se utiliza o bisturi monopolar o circuito elétrico é composto pela unidade eletrocirúrgica, pelo paciente e pelos eletrodos ativo (bisturi elétrico) e o de retorno (placa dispersiva). Desse modo, evidencia-se que o paciente faz parte do circuito (sistema) elétrico, pois este atua como condutor já que a corrente elétrica flui através do seu corpo (Bisinotto, et al., 2017).

Apesar dos avanços tecnológicos no campo da eletrocirurgia, ainda não se conseguiu uma garantia total de segurança máxima do uso desta tecnologia, uma vez que seu uso pode acometer o paciente (durante o período perioperatório) a inúmeras injúrias: queimaduras, fumaça cirúrgica, incêndios, além de outros menos divulgados ou até desconhecidos como perda, fuga ou desvio de corrente elétrica no campo operatório (Claudio, et al., 2017; Olímpio, Sousa \& Ponte, 2016; Tramontini, et al., 2016). 
Diante disso, se desperta a necessidade de mudança de atitude e estratégia para assegurar qualidade ao cuidado prestado tendo em vista que tais efeitos adversos poderiam ser evitados se a equipe que atua em centro cirúrgico tivesse maior conhecimento sobre dita tecnologia (Jones, et al., 2017).

Tendo em vista as necessidades e particularidades dos pacientes cirúrgicos, os cuidados de enfermagem perioperatório constituem uma cascata de ações indispensáveis para promover a segurança e o bem-estar do paciente e possui papel significativo nas ações de boas práticas no centro cirúrgico que visam à segurança e o reestabelecimento do cliente (Olímpio, Sousa \& Ponte, 2016).

Dessa forma, torna-se imprescindível buscar produções científicas acerca do cuidado de enfermagem frente à eletrocirurgia a fim de proporcionar a disseminação de conhecimentos sobre a temática e assim promover uma assistência de enfermagem segura.

Nessa perspectiva, o objetivo desse estudo foi analisar a produção científica publicada acerca do cuidado de enfermagem frente ao uso da eletrocirurgia, e identificar as contribuições destas para a qualidade da assistência.

\section{Metodologia}

O presente trabalho consiste em uma revisão integrativa que buscou analisar as produções científicas sobre os cuidados de enfermagem no contexto da eletrocirurgia e identificar as contribuições para a qualificação da assistência.

Esse tipo de pesquisa possibilita incluir estudos experimentais e não-experimentais para a compreensão do fenômeno a ser analisado. Com isso, permite além de aprofundar o conhecimento sobre a temática investigada, auxiliar integrar, relacionar as informações e apontar lacunas que precisam ser preenchidas por meio da realização de novas investigações para atualização e orientação de novas condutas profissionais (Ferreira, 2015; Pereira, et al., 2018).

Assim, a revisão integrativa consiste numa ferramenta para incorporar evidências na prática clínica. Desse modo, a pesquisa foi orientada pelas seis etapas que compõem esse tipo de estudo: 1) elaboração de uma questão norteadora 2) determinação dos critérios de inclusão e exclusão e busca na literatura; 3) delinear os procedimentos de busca; 4) avaliação dos trabalhos incluídos na revisão integrativa; 5) interpretação dos dados e; 6) a apresentação da revisão com síntese do conhecimento produzido (Mendes, Silveira \& Galvão, 2008).

Para a formulação da pergunta norteadora, foi utilizada a estratégia P.I.C.O a fim de estruturar a questão norteadora e possibilitar a coleta de informações precisas acerca do tema e extrair o máximo de evidências cientificas nas bases de dados (Tufanaru, et al., 2017). Sendo a seguinte: Quais as práticas baseadas em evidências acerca dos cuidados de enfermagem para o uso seguro da eletrocirurgia em pacientes no período intraoperatório? Com isso, após a escolha do tema e a formulação da pergunta de pesquisa, iniciou-se a busca nas bases de dados, para identificação dos estudos que serão incluídos na revisão.

Os critérios de inclusão para amostra dessa investigação foram teses, dissertações e os artigos originais, estudos que abordavam os cuidados de enfermagem aos pacientes submetidos a procedimentos ou intervenções em saúde com o uso da eletrocirurgia no período intraoperatório; escritos nos idiomas inglês, português e espanhol e publicados de janeiro de 2000 a dezembro de 2019.

Em relação aos critérios de exclusão optou-se por não incluir pesquisas incompletas e duplicados nas bases de dados consultadas, e os estudos conduzidos a respeito dos efeitos causados aos profissionais da saúde acerca do uso da eletrocirurgia, em animais "in vivo", estudos em "ex vivo" (em órgãos fora do corpo humano), artigos nota prévia, e os que abordam exclusivamente os custos na utilização desse recurso tecnológico. Além disso, foram excluídos os artigos que tinham como objetivo a comparação e eficácia clínica e a efetividade dos procedimentos ou técnicas utilizadas.

Para viabilizar as informações a respeito da utilização da eletrocirurgia foram realizadas buscas por produções científicas nas bases de dados: MEDLINE® via PubMed®, Literatura Latino-Americana e do Caribe em Ciências da Saúde 
(LILACS). Também se realizou pesquisa no portal da Biblioteca Virtual da Saúde - Enfermagem e Scientific Electronic Library Online (SciELO) e no portal Google acadêmico.

Com o objetivo de uniformizar a terminologia, organizar, recuperar e disseminar as informações para pesquisa na área das ciências da saúde foram desenvolvidos vocabulários controlados (descritores), tais como Descritores em Ciências da Saúde (DeCS) e Medical Subject Headings (MeSH). O vocabulário controlado MeSH foi publicado pela National Library of Medicine com a finalidade de padronizar o uso de uma mesma terminologia na América Latina no idioma inglês. Devido isso, o MeSH foi utilizado nesse estudo já que é um recurso obrigatório para a indexação e coleta de informações acerca dos artigos publicados na base de dados MEDLINE® via PubMed®. Para a pesquisa nas outras bases de dados, bibliotecas e Google acadêmico foi utilizado DeCS, entretanto como são dois recursos de pesquisa diferentes para não ocorrer divergência na coleta de dados foram pesquisadas as terminologias da área da saúde similares usadas nesse estudo.

Ressalta-se que a estratégia de busca foi arquitetada de maneira minuciosa para identificação dos estudos publicados nas bases de dados, bibliotecas e portais. Para isso, uma busca inicial foi realizada utilizando-se descritores controlados identificados nos MeSH e após a mesma pesquisa com os mesmos vocábulos no DECs.

Assim, com o recurso MeSH e DECs, os vocábulos controlados utilizados foram: "Eletrocirurgia", "Cuidados de Enfermagem", "Período Intraoperatório", "Eletrocoagulação", e "Segurança do Paciente”. A estratégia de busca ocorreu a partir de suas diferentes combinações, utilizando-se o operador booliano AND nos idiomas português quando utilizado o DeCS e inglês quando MeSH conforme recomendação dos portais de pesquisa.

No entanto, mesmo com conferência dos termos iguais no MeSH e DeCs, observou que a utilização de descritores padronizados, conforme proposta do vocabulário controlado, ainda não foi adotada universalmente. Encontra-se erros de indexação, utilização de descritores não-controlados apresentados como palavras-chave nos artigos publicados, desse modo evidencia uma ausência de padronização absoluta da linguagem cientifica na área da saúde

Para que se pudesse mergulhar na produção científica encontrada na literatura e realizar uma estratégia de busca bem definida, sentiu-se a necessidade de utilizar palavras-chaves, além dos descritores padronizados. Devido isso, foi realizada uma análise e adesão dos vocabulários não controlados denominados como sinônimo ou palavra-chave. Assim, após várias mudanças na composição/agrupamento das palavras-chaves, averiguou-se que as que melhor atenderam este desenho metodológico foram: "Dispositivos eletrocirúrgicos", "Dispositivos geradores de energia", "Enfermagem Perioperatória", "Eletrocautério", "Ondas de radiofrequência".

Desse modo, a estratégia de busca nesta pesquisa ocorreu a partir de suas diferentes combinações conforme apresentados no Quadro 1, utilizando-se o operador booliano AND no idioma português quando utilizado o DeCS e inglês quando MeSH conforme recomendação dos portais de pesquisa.

Assim, a busca nas bases de dados possibilitou encontrar os artigos e realizar a seleção dos trabalhos que compuseram (a partir da leitura dos títulos e, em seguida, dos resumos) a amostra deste estudo. Posteriormente, foram realizadas a leitura na íntegra e análise dos trabalhos, após a averiguação dos que atendiam os critérios de elegibilidade determinados nesta investigação.

Cabe ressaltar que os artigos selecionados foram detalhadamente analisados e classificados em relação ao nível de evidência conforme proposto por Melnyk \& Fineout-Overholt (2011) onde possibilitou categorizar as publicações de acordo com a metodologia utilizada no estudo como: nível I corresponde aos estudos provenientes de revisão sistemática ou metanálise de ensaios clínicos randomizados controlados ou oriundos de diretrizes clínicas baseadas em revisões sistemáticas de ensaios clínicos randomizados controlados; nível II estudos decorrentes de ensaio clínico randomizado controlado bem delineado; nível III baseado em evidências de ensaios clínicos bem delineados sem randomização; nível IV estudos de coorte e de caso-controle bem delineados; nível $\mathrm{V}$ evidências originárias de revisão sistemática e integrativa de estudos descritivos 
quantitativos e qualitativos; nível VI estudo descritivo quantitativo ou qualitativo; e o nível VII corresponde a estudos de opinião de autoridades e/ou relatório de comitês de especialistas obtidas.

Quadro 1: Estratégia de busca das diferentes combinações utilizada nas bases de dados, biblioteca e portais usando os vocábulos controlados (descritores) e o vocabulários não controlados (palavras-chave). Maceió, AL, Brasil, 2020.

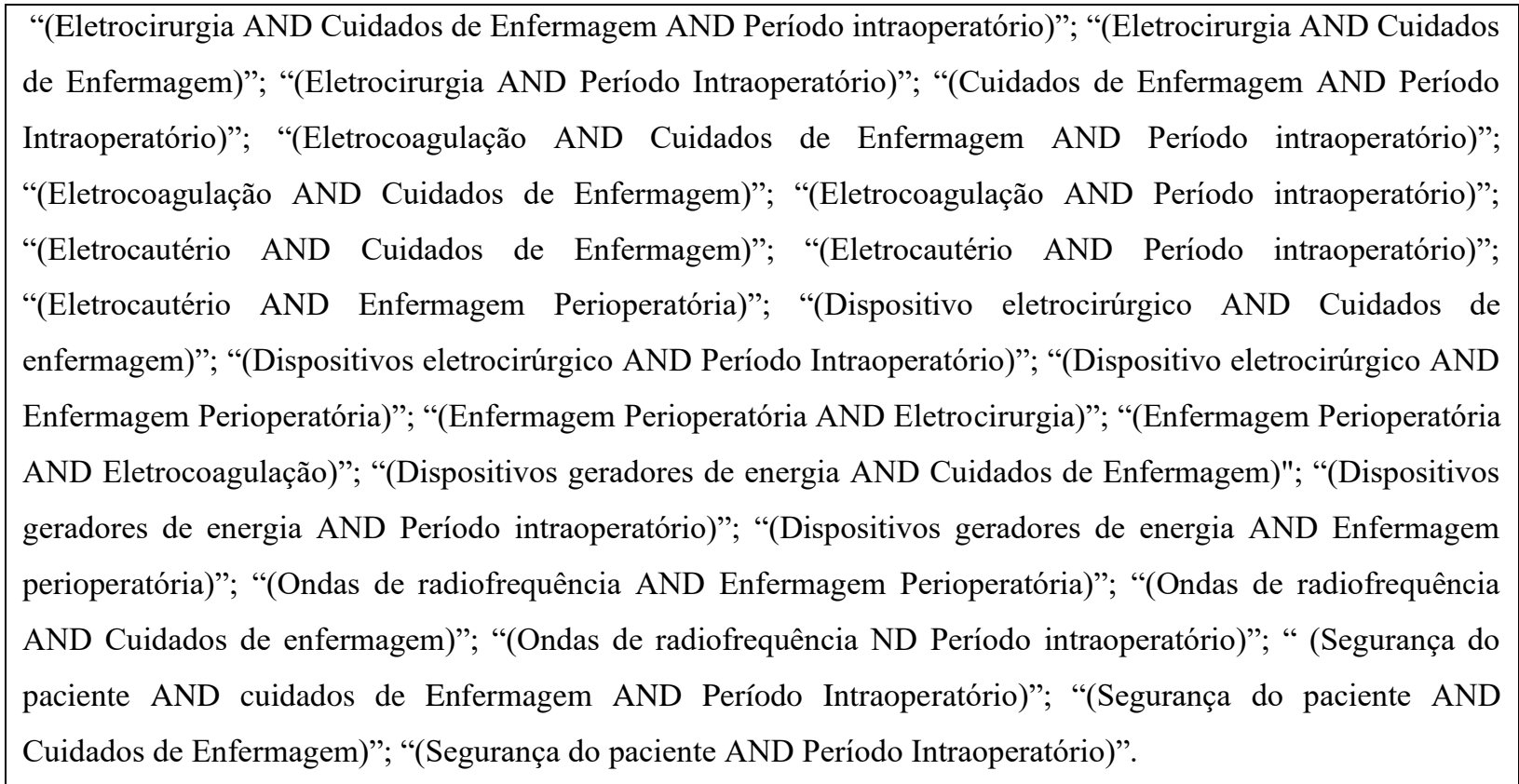

Fonte: Autores.

Após a análise dos artigos, foram construídos gráficos e tabelas com os principais resultados e realizada a discussão desses, mediante referencial teórico.

\section{Resultados}

O levantamento das publicações científicas, conforme descritos na Tabela 1, nas bases de dados LILACS e MEDLINE® via PubMed®, nas bibliotecas BVS-Enfermagem e Scielo, e no portal Google Acadêmico foi realizado, concomitantemente, entre os meses de agosto e outubro de 2020. 
Tabela 1: Distribuição dos estudos encontrados por base de dados, biblioteca e portais. Maceió, AL, Brasil, 2020.

\begin{tabular}{|c|c|c|c|c|c|}
\hline & SCIELO & LILACS & BVS-ENF & PUBMED & $\begin{array}{c}\text { GOOGLE } \\
\text { ACADÊMICO }\end{array}$ \\
\hline LOCALIZADOS (L) & 257 & 127 & 5.079 & 19.854 & 130.383 \\
\hline $\begin{array}{c}\text { PERMANECERAM } \\
\text { APÓS LEITURA DE } \\
\text { TÍTULO (T) }\end{array}$ & 3 & 9 & 65 & 136 & 162 \\
\hline $\begin{array}{l}\text { PERMANECERAM } \\
\text { APÓS LEITURA DE } \\
\text { RESUMO }(R)\end{array}$ & 0 & 7 & 59 & 107 & 150 \\
\hline $\begin{array}{c}\text { CRITÉRIOS DE } \\
\text { ELEGIBILIDADE (E) }\end{array}$ & 0 & 7 & 39 & 49 & 114 \\
\hline $\begin{array}{c}\text { ESTUDOS EXCLUÍDOS } \\
\text { POR ESTAREM } \\
\text { DUPLICADOS }\end{array}$ & 0 & 4 & 26 & 37 & 101 \\
\hline AMOSTRA FINAL & 0 & 3 & 13 & 12 & 13 \\
\hline
\end{tabular}

Fonte: Dados da pesquisa (2020).

Por meio da Tabela 1, percebe-se que foram localizadas 155.700 publicações, no entanto, após análise dos títulos dos estudos permaneceram 375 publicações para etapa posterior. A seguir, foi realizada a leitura dos resumos que possibilitou uma pré-seleção de 323 estudos para análise dos critérios de inclusão. Destes, 224 estudos seguiram para análise detalhada e coleta de dados, onde 168 publicações foram excluídas por estarem repetidas com os diferentes cruzamentos de descritores e palavras-chaves durante a pesquisa na mesma base de dados portais, e bibliotecas digitais.

Dessa forma, ao excluir os artigos repetidos (obtidos com a realização dos diversos cruzametos descritos), obteve-se as seguintes conclusões: ausência de pesquisa científica na biblioteca SCIELO acerca dos cuidados de enfermagem para o uso seguro da eletrocirurgia em pacientes no período intraperatório; havia 3 (três) estudos na LILACS; 13 na BVSEnfermagem; 12 na MEDLINE® via PubMed®; 13 no Google Acadêmico resultando, assim, em 41 estudos para leitura na íntegra conforme apresentado na Tabela 1. No entanto, foi constatado a duplicação de 17 publicações, dessa maneira a amostra final foi composta por $24(100 \%)$ estudos.

Verificou-se que dos 24 estudos selecionados 9 (37,5\%) foram publicados apenas no Google Acadêmico, dois $(8,3 \%)$ na PUBMED, um (4,2\%) na BVS-Enfermagem, e os outros 12 (50\%) estudos foram localizados concomitantemente nas bases de dados pesquisadas.

Em relação ao ano que mais teve publicações foi observado que cinco (20,8\%) foram publicadas em 2017. Quanto ao periódico de publicação dos estudos, cinco (20,8\%) foram indexados em AORN Journal, três (12,5\%) publicações foram na Revista SOBECC. O Quadro 2 evidencia que os demais $(66,7 \%)$ periódicos tiveram apenas uma publicação cada.

Sobre os tipos de publicação, no que diz respeito à abordagem metodológica, verificou-se que: 9 (37,5\%) são de estudos de revisão integrativa; 5 (20,8\%) relatos de caso; 3 (12,5) descritivos; pesquisa exploratória e quase-experimental ambas com um (4,2\%) estudo cada. Constatou-se ausência do delineamento metodológico em 5 (20,8\%) estudos, conforme Quadro 2. 
No que se refere ao nível da evidência científica dos trabalhos que compuseram esta amostra, percebeu-se que 9 (55,6\%) estudos têm o nível de evidência 5 (NE-V); 9 (55,6\%) estudos com nível de evidência 6 (NE-VI); um (4,2\%) estudo com nível de evidência 3 (NE-III).

Quadro 2: Distribuição dos estudos segundo autor, ano, periódico, tipo de estudo e nível de evidência. Maceió, AL, Brasil, 2020 .

\begin{tabular}{|c|c|c|}
\hline $\begin{array}{c}\text { Identificação do artigo - } \\
\text { Autores e Ano }\end{array}$ & Periódico indexado & Tipo de estudo e nível de evidência \\
\hline A1-Brito \& Galvão, (2009). & Rev Gaúcha Enferm & Revisão integrativa. NE-V \\
\hline A2-Olímpio, Sousa \& Ponte, (2016). & Rev SOBECC & Revisão integrativa. NE-V \\
\hline $\begin{array}{l}\text { A3-Parra, Giannasttasio \& Diniz, } \\
\text { (2012). }\end{array}$ & Rev SOBECC & $\begin{array}{l}\text { Descritiva- exploratória, quantitativa. } \\
\text { NE-VI }\end{array}$ \\
\hline A4-Sandes, et al., (2019). & Rev SOBECC & Revisão integrativa. NE-V \\
\hline A5-González, et al., (2009). & Rev Esp Anestesiol. Reanim. & Relato de caso NE-VI \\
\hline A6-Eder, (2017). & AORN Journal. & Relato de caso.NE-VI \\
\hline A7-Spruce \& Braswell, (2011). & AORN Journal. & Ausente \\
\hline A8-Morton, (2012). & AORN Journal. & Revisão integrativa. NE-V \\
\hline A9-Naik, et al., (2017). & Journal of Electrocardiology & Relato de caso. NE-VI \\
\hline A10-O'Riley, (2010). & Open Learning Zone & Estudo descritivo. NE-VI \\
\hline A11-Aorn, (2005). & AORN Journal & Ausente \\
\hline A12-Wicker, (2000) & $\begin{array}{l}\text { British Journal Of Perioperative } \\
\text { Nursing }\end{array}$ & Estudo descritivo. NE-VI \\
\hline A13-Aorn, (2004). & AORN Journal & Ausente \\
\hline A14-Diccini \& Nogueira, (2008). & Rev Bras Enferm & Revisão integrativa. NE-V \\
\hline A15-Ulmer, (2002) & Plastic Surgical Nursing & Ausente \\
\hline A16-Brito, (2007). & Biblioteca digital USP & Revisão integrativa. NE-V \\
\hline A17-Almeida, (2019). & Biblioteca digital USP & Quase- experimento. NE- III \\
\hline A18-Silva, (2017). & $\begin{array}{l}\text { Repositório Institucional } \\
\text { Faculdade de Educação e Meio } \\
\text { Ambiente -FAEMA }\end{array}$ & Revisão integrativa. NE-V \\
\hline A19-Piccoli, et al., (2005). & $\begin{array}{l}2^{\circ} \text { Seminário Nacional Estado e } \\
\text { Políticas Sociais No Brasil } \\
\text { (UNIOESTE) }\end{array}$ & Ausente \\
\hline A20-Cruz, et al., (2014). & $\begin{array}{l}\text { XXIV Congresso Brasileiro de } \\
\text { Engenharia Biomédica }\end{array}$ & Pesquisa exploratória. NE-VI \\
\hline A21-Gomide, et al., (2011) & Rev Bras Ortop & Relato de caso. NE-VI \\
\hline A22-Bisinotto, et al., (2017). & Rev Bras Anestesiol & Relato de caso. NE- VI \\
\hline A23-Martins, (2014). & Percursos & Revisão integrativa. NE-V \\
\hline A24-Xavier, (2017). & $\begin{array}{l}\text { Repositório Institucional Hosp } \\
\text { Geral de Fortaleza }\end{array}$ & Revisão integrativa. NE-V \\
\hline
\end{tabular}


Fonte: Dados da pesquisa (2020).

O Quadro 2 destaca que os anos de 2001, 2003, 2006, 2013 e 2018 não tiveram publicações relacionados ao objeto desta revisão publicados. Os estudos que compuseram a amostra final mostraram que o tema abordado nesta investigação (Cuidado de Enfermagem frente ao uso da eletrocirurgia) não teve publicações apenas nas revistas de enfermagem perioperatória e, também, essa temática não foi exclusiva de pesquisas na área da saúde. Outro fator observado, é que os trabalhos publicados não possuem nível alto de evidência científica.

O Quadro 3 apresenta os fatores elencados nas pesquisas publicadas que se relacionaram ao uso da unidade de eletrocirurgia. Ao analisar a essência dos conteudos constatou-se que 62,5\% dos estudos abordaram o conhecimento da equipe de enfermagem sobre o uso da eletrocirurgia e, é importante evidenciar, que 37,5\% apresentaram como resultados para a ocorrência de eventos adveros as possíveis complicações decorrentes do uso inadequado da unidade de eletrocirurgia.

Outro aspecto identificado em aproximadamente $71 \%$ das publicações da amostra deste estudo foi a ocorrência de queimaduras em decorrência do mau uso da eletrocirurgia. Além disso, observou-se que 37,5\% dos estudos descreveram as injúrias ocasionadas pelo uso errôneo de uma unidade eletrocirurgica. E, em $25 \%$ dos estudos, foi retratado a incidência de acidentes com fogo quando envolvia o uso da referida tecnologia.

Constatou-se, também, que 37,5\% das publicações expõem alguma recomendação relacionada ao uso da eletrocirurgia, e $25 \%$ destas discorrem sobre o funcionamento da unidade eletrocirurgica. Outro resultado encontrado, em 45,8\% das investigações que compusseram a amostra deste estudo, foi em relação a necessidade de se adotar medidas de segurança e os cuidados relacionados com a tecnologia empregada. Apenas 20,8\% das publicações mencionaram a descarga de energia perdida da unidade eletrocirúrgica. Ainda, foi possível constatar em apenas 12,5\% estudos foi mencionada a necessidade de cuidados específicos com os pacientes portadores de marcapasso que utilizam bisturi elétrico.

Observou-se que aproximadamente $60 \%$ dos artigos referiram a necessidade do paciente, também, adotar medidas de segurança para minimizar os potenciais riscos oriundos da unidade eletrocirurgia. Apenas 33,3\% das publicações abordaram o papel do enfermeiro perioperatório para favorecer, garantir a prevenção e a segurança no uso da eletrocirurgia.

Evidencia-se, ainda, que em apenas $12,5 \%$ dos estudos referiram a necessidade de educação e informações aos pacientes sobre as práticas seguras para o uso de eletrocirurgia. Além disso, observou-se que menos de $20 \%$ dos trabalhos mencionaram a importância da comunicação entre os membros da equipe cirúrgica como recurso que favorece o uso seguro da eletrocirurgia.

Outro aspecto citado em 37,5\% das investigações que compuseram esta amostra foi a ocorrência de eventos adversos devido ao mau uso e má manutenção da unidade eletrocirúrgica. Em pouco mais de $37 \%$ dos estudos referiram a necessidade de conhecer os principios, funcionamento dos diferentes equipamentos para o uso seguro da eletrocirurgia. E em 45,8\% das publicações foram mencionadas as recomendacões e cuidados com o posicionamento e uso seguro da placa eletrodispersiva. 
Quadro 3: Síntese dos aspectos relacionados ao uso da unidade de eletrocirurgia. Maceió, AL, Brasil, 2020.

\begin{tabular}{|c|c|}
\hline $\begin{array}{c}\text { Sínteses dos aspectos relacionados ao uso da unidade de } \\
\text { eletrocirurgia encontrados nos artigos }\end{array}$ & Identificação dos Artigos \\
\hline $\begin{array}{l}\text { I-Aborda o conhecimento da equipe de enfermagem acerca do } \\
\text { uso da eletrocirurgia. }\end{array}$ & $\begin{array}{l}\text { A1; A2; A3, A6; A7; A8; A9; A11; } \\
\text { A12; A13;A15; A16; A17; A18; A22 }\end{array}$ \\
\hline $\begin{array}{l}\text { II-Apresenta possíveis complicações decorrente do uso da } \\
\text { unidade de eletrocirurgia. }\end{array}$ & $\mathrm{A} 1, \mathrm{~A} 2, \mathrm{~A} 5, \mathrm{~A} 6, \mathrm{~A} 7, \mathrm{~A} 9, \mathrm{~A} 16, \mathrm{~A} 23 ; \mathrm{A} 24$ \\
\hline III-Expõe recomendações acerca do uso da eletrocirurgia. & A1;A3;A7;A9; A10; A16; A19; A20; A22 \\
\hline IV-Discorre sobre o funcionamento da unidade eletrocirurgica & A5;A6;A9; A10; A19;A20 \\
\hline V-Identifica a ocorrência de queimaduras & $\begin{array}{l}\text { A1; A4; A5; A6; A7; A8; A9; A10; A11; A12; } \\
\text { A13; A14; A16; A17; A21; A22; A24 }\end{array}$ \\
\hline $\begin{array}{l}\text { VI-Retrata a incidência de acidentes com fogo quando envolvia } \\
\text { o uso da eletrocirurgia. }\end{array}$ & A2; A4; A5; A7; A23; A24 \\
\hline $\begin{array}{l}\text { VII-Medidas de segurança e os cuidados relacionados com a } \\
\text { tecnologia empregada }\end{array}$ & $\begin{array}{l}\text { A2; A3; A8; A9; A10;A11; A13; A16; A21; } \\
\text { A23; A24 }\end{array}$ \\
\hline $\begin{array}{l}\text { VIII- Descrições de injúrias ocasionada pelo uso da unidade } \\
\text { eletrocirugia }\end{array}$ & A2; A4; A5; A6; A7; A9; A14; A22; A24 \\
\hline $\begin{array}{l}\text { IX- Refere as necessidades de medidas de segurança do } \\
\text { paciente para minimizar os potenciais riscos da unidade } \\
\text { eletrocirurgia. }\end{array}$ & $\begin{array}{l}\text { A1; A2; A3; A7; A8; A9; A10; A11; A13; A16; } \\
\text { A17; A19; A22; A23 }\end{array}$ \\
\hline $\begin{array}{l}\text { X- Abordam o papel do enfermeiro perioperatório para } \\
\text { favorecer e garantir a prevenção e segurança no uso da } \\
\text { eletrocirurgia. }\end{array}$ & A1; A2; A7; A9; A11;A13 A14; A16 \\
\hline $\begin{array}{l}\text { XI-Cuidados específicos com os pacientes portadores de } \\
\text { marcapasso ao utiliza a eletrocirurgia. }\end{array}$ & A3; A7; A9 \\
\hline $\begin{array}{l}\text { XII-Mencionam a descarga de energia perdida da unidade } \\
\text { eletrocirúrgica }\end{array}$ & A6; A7; A12; A23; A24 \\
\hline $\begin{array}{l}\text { XIII-Refere a educação e informações ao paciente sobre as } \\
\text { práticas seguras para o uso de eletrocirugia }\end{array}$ & A8; A9; A24 \\
\hline $\begin{array}{l}\text { XIV- Refere as causas de eventos adversos devido ao mau uso e } \\
\text { má manutenção da unidade eletrocirúrgica }\end{array}$ & A5; A6; A7; A10; A15; A16; A18; A23; A24 \\
\hline $\begin{array}{l}\text { XV-Refere a importãncia da comunicação entre a equipe } \\
\text { cirúurgica no uso da eletrocirurgia. }\end{array}$ & A2;A6; A8; A23 \\
\hline $\begin{array}{l}\text { XVI-Refere a necessidade de conhecer os princípios, } \\
\text { funcionamento dos diferentes equipamento para o uso seguro da } \\
\text { eletrocirurgia }\end{array}$ & A1; A3; A11;A12;A13; A15; A18; A20; A23 \\
\hline $\begin{array}{l}\text { XVII- Recomendacões e cuidados com o posicionamento e uso } \\
\text { da placa eletrodispersiva }\end{array}$ & $\begin{array}{l}\text { A3; A10; A11;A12; A13; A16; A } 17 ; \text { A } 18 ; \text { A20; } \\
\text { A21; A22 }\end{array}$ \\
\hline
\end{tabular}

Fonte: Dados da pesquisa (2020). 
O Quadro 3 evidencia as particularidades que os estudos apresentam em relação ao uso do bisturi elétrico e algumas medidas que devem ser adotadas para prevenir a ocorrência de danos evitáveis e garantir o uso seguro da unidade eletrocirurgia no intraoperatório.

Os estudos mostraram que a identificação dos possíveis eventos adversos resultantes do mau uso da eletrocirurgia nos procedimentos cirúrgicos é um desafio possível de ser superado pela equipe de enfermagem por meio do domínio da tecnologia e planejamento da assistência.

\section{Discussão}

A unidade eletrocirúrgica tem sido muito aplicada em procedimentos cirúrgicos, pois ao utilizar-se uma corrente elétrica de alta frequência possibilita cortar o tecido e controlar o sangramento ao provocar coagulação. No entanto, mesmo sendo considerado um recurso útil e efetivo, requer vigilância constante para prevenir a ocorrência de eventos adversos e garantir padrões de segurança durante a aplicação dessa tecnologia (Almeida, 2019; Bisinotto, et al., 2017).

Esta afirmação tem amparo nas orientações da Organização Mundial de Saúde (OMS), que desde o ano de 2008, afirma que a segurança do paciente pode ser alcançada por meio de três ações complementares: evitar a ocorrência dos eventos adversos; torná-los conhecidos caso ocorram e minimizar seus efeitos com intervenções eficazes.

Henrique, Costa \& Lacerda (2016) em uma revisão integrativa relataram estudos que apontam a ocorrência de lesões na pele dentre os principais erros e fragilidades da assistência de enfermagem que colocam em risco a segurança do paciente no ambiente intraoperatório. Afirmaram, ainda, que o bloco cirúrgico é o local do hospital onde acontece grande parte dos eventos adversos à saúde do paciente devido aos inúmeros fatores e complexidade que estão ligados às intervenções cirúrgicas.

Outro aspecto identificado que favorece as boas práticas na assistência em saúde é a participação do paciente na adesão às medidas de segurança. Entretanto, para Afonso, et al., (2010) nem sempre existe a cooperação do cliente às medidas preventivas dos riscos que podem ser causadas pela unidade eletrocirúrgica, declarando motivação pela temática estudada devido à recursa de uma paciente em retirar os anéis metálicos de seus dedos devido à incapacidade de o fazer sem danificá-los.

Sob a égide desse acontecimento, entende-se que para uma melhor eficácia das boas práticas no uso da eletrocirurgia faz-se necessário incluir o paciente no seu processo de cuidado, orientando a respeito das medidas de segurança que ele pode aderir. Assim, para Wegner, et al., (2016) e Olímpio, Sousa \& Ponte (2016) promover a educação do paciente é um aspecto essencial para garantir práticas seguras na assistência em saúde, em especial no uso da eletrocirurgia.

Desse modo, para que haja uma melhor incorporação das estratégias necessárias durante a intervenção cirúrgica é fundamental a participação do paciente cirúrgico para enfrentar e decidir, de forma consciente e informada, acerca das práticas seguras em prol de uma assistência de qualidade. Logo, é atribuição do enfermeiro informar o paciente como ele pode participar das ações de seguranças que devem ser adotadas no uso da unidade eletrocirúrgica (Henriques, Costa \& Lacerda, 2016; Almeida, 2019).

Neste sentido, emerge a imputação de se ter uma enfermagem alinhada às necessidades dos pacientes e com capacidade de prevenir as diversas injúrias que ocorrem, principalmente, nas salas de operação. Assim, se torna notória a criação de ferramentas (com uso de tecnologias leves) que auxiliem as práticas diárias da enfermagem para a obtenção de um cuidado seguro e de excelência.

Almeida (2019) informa que a incorporação de boas práticas na sala operatória favorece a efetividade dos cuidados e o seu gerenciamento de modo seguro, mas evidencia que esta incorporação ainda não se tornou uma realidade global. 
Diante dessa lacuna, percebe-se que a falta de preparo pré-operatório eficaz e/ou a realização incompleta dos cuidados necessários podem ser desencadeadores de incidente durante o período intraoperatório em decorrência da falta de medidas preventivas e promoção efetiva para diminuir os danos e garantir segurança no procedimento cirúrgico (Gutierres, et al., 2018). Corroborando com esse entendimento o estudo de Stuchi, Cheble \& Matayoshi (2020) destacou a necessidade de identificar ainda no período pré-operatório os potenciais riscos ou as possíveis causas de eventos adversos na sala cirúrgica e, ainda, descreveu a importância de implementar um plano de cuidado com ações para reduzir os riscos durante o uso da unidade eletrocirúrgica.

Assim, destaca-se, também, que a consulta de enfermagem e a elaboração de um plano de cuidados voltado para cada paciente cirúrgico, respeitando sua individualidade, são necessários para a garantia da qualidade na assistência no período perioperatório.

Embora seja uma prática rotineira durante os procedimentos cirúrgicos, e mesmo que os avanços tecnológicos tenham reduzido a ocorrência de eventos adversos relacionados ao uso de unidades eletrocirúrgicas, não se pode esquecer de que o corpo do paciente é parte integrante do circuito elétrico quando utilizado o bisturi elétrico monopolar e, por isso, quando ocorrem falhas e inconformidades no circuito da corrente elétrica dispensada pode causar diversos danos (Farias, et al., 2019).

Nessa perspectiva, estudos apontam que é imperativo ao enfermeiro perioperatório o conhecimento técnicocientífico sobre o uso da eletrocirurgia e, ainda, afirmam que este profissional tem papel fundamental e indispensável nas ações que eliminem ou diminuam os riscos potenciais decorrentes da utilização desse recurso tecnológico (Brito \& Galvão, 2009; Olímpio, Sousa \& Ponte, 2016; Soares, Sousa \& Castro, 2016).

Além disso, a equipe de enfermagem possui posição privilegiada no que concerne ao planejamento do cuidado, possibilitando a partir do conhecimento técnico-científico avaliar e prevenir possíveis complicações decorrentes dos recursos utilizados no ato cirúrgico (Panzetti, et al., 2020)

Contudo, para que a unidade eletrocirúrgica seja usada de maneira que minimize a potencial ocorrência de eventos adversos deve-se buscar estratégias que favoreçam a diminuição dos riscos e a efetividade da intervenção cirúrgica, garantindo a segurança dos pacientes.

Desse modo, ressalta a importância de usar a corrente elétrica com pulsos curtos, intermitentes e irregulares nos menores níveis possíveis de energia minorando os efeitos hemodinâmicos durante o uso da eletrocirurgia. Portanto, o uso do bisturi elétrico monopolar deve ser cauteloso para evitar corte e coagulação excessivos (Rapsang \& Bhattacharyya, 2014; Xavier, 2017; Alves Júnior, et al., 2020).

Foi percebido que a presença de objetos metálicos como os adornos ou até os eletrodos de monitoramento cardíaco, a colocação inadequada da placa dispersiva, assim como o contato do paciente com superfície metálica da mesa cirúrgica podem favorecer a concentração ou a fuga de corrente de energia da unidade eletrocirúrgica provocando lesões irreversíveis nos pacientes.

Por esse motivo, acredita-se que ao realizar um planejamento das ações e utilizar uma lista de verificação auxiliará de forma mais adequada as condutas no uso da unidade eletrocirúrgica e proporcionará um plano de cuidados de enfermagem efetivo e satisfatório ao direcionar as práticas seguras e as necessidades particularidades dos clientes.

\section{Considerações Finais}

Os resultados deste estudo sinalizam uma produção científica ainda em progressão, e apontam que a equipe de enfermagem se encontra dentre os profissionais que desempenham um importante e indispensável papel no cuidado acerca da eletrocirurgia no período intraoperatório. 
Portanto, é nítida a importância de incluir medidas de segurança para garantir intervenções adequadas ao paciente cirúrgico durante a utilização da eletrocirúrgica. Conclui-se, ainda, que há uma necessidade de conhecer os princípios e funcionamento dos diferentes equipamentos, e com isso minimizar e eliminar os possíveis eventos adversos decorrente do uso desse recurso tecnológico na assistencia em saúde.

Há necessidade ainda de pesquisas futuras, viabilizadas por outros métodos cientificos, acerca do conhecimento da equipe de enfermagem sobre o funcionamento e uso seguro da eletrocirurgia com incorporação de medidas de seguranças que possam contribuir em um direcionamento amplo, adequado e eficaz dos cuidados de enfermagem no perioperatório.

\section{Referências}

Afonso, C. T., Silva, A. L., Fabrini, D. S., Afonso, C. T., Côrtes, M. G. W., \& Sant'anna, L. L. (2010). Riscos do uso do eletrocautério em pacientes portadores de adornos metálicos. ABCD Arq Bras Cir Dig, 23(3), 183-186.

Almeida, C. L. (2019). Avaliação de intervenções educativas na prática e conhecimento da equipe médica e de enfermagem no uso de eletrocirurgia. http://www.teses.usp.br/teses/disponiveis/22/22132/tde-22102019-211321/ >.

Alves Júnior, L. C., Sousa, B. B., Zacarias, V. L. B., \& Germano, A. R. (2020). Lipectomia oral: relato de complicação cirúrgica mediata. Research, Society and Development, 9 (10), e4949108921. doi: https://doi.org/10.33448/rsd-v9i10.8921

Association of periOperative Registered Nurses Recommended Practices Committee. (2004). Recommended practices for electrosurgery. AORN J, 79(2):432-4, 437-42, 445-50. 10.1016/s0001-2092(06)60620-0.

Association of periOperative Registered Nurses Recommended Practices Committee. (2005). Práticas Recomendadas para Eletrocirurgia. AORN J, 81 (03). https://doi.org/10.1016/S0001-2092(06)60447-X

Bisinotto, F. M. B., Dezena, R. A., Martins, L. B., Galvão, M. C., Sobrinho, J. M., \& Calçado, M. S. (2017). Queimaduras relacionadas à eletrocirurgia Relato de dois casos. Revista Brasileira de Anestesiologia, 67(5), 527-534.

Brito, M. F. P., \& Galvão, C. M. (2009). Os cuidados de enfermagem no uso da eletrocirurgia. Revista Gaúcha de Enfermagem, 30 (2): $319-327$. http://seer.ufrgs.br/RevistaGa uchadeEnfermagem/article/view/5733

Brito, M. F. P. (2007). Enfermagem: evidências para o cuidado de enfermagem. https://teses.usp.br/teses/disponiveis/22/22132/tde15102007145726/publico/Mariadefatimapaivabrito.pdf

Claudio, C. V., Ribeiro, R. P., Martins, J. T., Marziale, M. H. P., Solci, M. C. \& Dalmas, J. C. (2017). Polycyclic aromatic hydrocarbons produced by electrocautery smoke and the use of personal protective equipment. Revista Latino-Am. Enfermagem, 25 (2853). http://dx.doi.org/10.1590/15188345.1561.2853.

Cruz, G. T., Ribeiro, L. R. F., Lima, B. F. S., Pereira, D. M., \& Rodrigues, M. A. B. (2014). Riscos e causas de queimaduras em pacientes quando submetidos a procedimentos cirúrgicos com o uso de unidade eletrocirúrgica. https://www.canal6.com.br/cbeb/2014/artigos/cbeb2014_submission_758.pdf

Diccini, S., \& Nogueira, A. C. (2008). Remoção do piercing no perioperatório: [revisão]. Revista Bras. Enferm, 61(1), 85-90. <http://www.scielo.br/scielo.php?script=sci_arttext\&pid=S0034-71672008000100013\&lng=en\&nrm=iso>.

Duarte, S. C. M., Stipp, M. A. C., Silva, M. M., \& Oliveira, F. T (2015). Eventos adversos e segurança na assistência de enfermagem. Revista Bras. Enferm, 68(1), 144-154. doi: https://doi.org/10.1590/0034-7167.2015680120p

Eder, S. P. (2017). Guideline Implementation: Energy-Generating Devices, Part 1-Electrosurgery. AORN J,105(3), 300-310. 10.1016/j.aorn.2017.01.004. 
Farias, I. P., Almeida, T. G., Lopes, R. F., Torres, B. A. T., Silva, R. R. S. M., Trindade, R. F. C., Macedo, J. K. S. S., \& Vasconcelos, E. L. O uso seguro de eletrocirurgia. Revista enferm. UFPE on line,13(2), 538-541.

Ferreira, C. A. L. (2015). Pesquisa quantitativa e qualitativa: perspectivas para o campo da educação. Revista Mosaico, $173-182$. seer.pucgoias.edu.br/index.php/mosaico/article/download/4424/2546

Gomide, L. C., Santos, C. E. R. G., Pereira, C. J., Carvalho, L. C. C, Queiroz, S. A. S., \& Cunha, L. R., et al. (2011). Queimadura de terceiro grau na região da placa eletrocirúrgica durante artroscopia de ombro: relato de caso. Revista Bras. Ortop, 46(1), 91-93. http://dx.doi.org/10.1590/S010236162011000100017

González, A. V., Valién, J. L., Armijo, J. V., Lobera, C. (2009). Ignición del tubo orotraqueal inducida por electrocauterio durante una traqueostomia. Revista Española de Anestesiología y Reanimación, 56 (1), 47-49. 10.1016/S0034-9356(09)70320-2

Gutierres, L. S., Santos, J. L. G., Peiter, C. C., Menegon, F. H. A., Sebold, L. F., \& Erdann, A. L. (2018). Boas práticas para segurança do paciente em centro cirúrgico: recomendações de enfermeiros. Revista Bras. Enferm, 71(Suppl 6), 2775-2782. https://doi.org/10.1590/0034-7167-2018-0449

Henriques, A. H. B., Costa, S. S., Lacerda, J. S. (2016). Assistência de enfermagem na segurança do paciente cirúrgico: revisão integrativa. Cogitare Enfermagem, 21 (4), 01-09.

Jones, S. B., Munro, M. G., Feldman, L. S., et al (2017). Uso fundamental da energia cirúrgica (FUSE): Um programa educacional essencial para a segurança da sala de cirurgia. Perm J, 21, (16-050). doi: https://doi.org/10.7812/TPP/16-050.

Martins, L. (2014). Prevenção do Incêndio cirúrgico. Percursos, 30. https://comum.rcaap.pt/bitstream/10400.26/9275/1/Revista\%20 Percursos\%20n30_Preven\%C3\%A7\%C3\%A3o\%20do\%20Inc\%C3\%AAndio\%20Cir\%C3\%BArgico.pdf

Melnyk, B. M., \& Fineout -Overholt, E. (2011). Evidence-based practice in nursing and health care: A guide to best practice. Philadelphia: Lippincott, Williams \& Wilkins.

Mendes, K. D. S., Silveira, R. C. C. P., \& Galvão, C. M. (2008). Revisão integrativa: método de pesquisa para a incorporação de evidências na saúde e na enfermagem. Texto \& Contexto Enfermagem, 17(4), 758-764.

Morton, P. J. (2012). Implementing AORN recommended practices for minimally invasive surgery: part I. AORN J, 96(3):295-312. 10.1016/j.aorn.2012.06.009

Naik, B. N., Luthra, A., Dwivedi, A., \& Jafra, A. (2017). Artifactual ECG changes induced by electrocautery in a patient with coronary artery disease. J Electrocardiol, 50(4):531-533. 10.1016/j.jelectrocard.2017.03.005. .

Nascimento, J. C., Draganow, P. B. (2015). História da qualidade em segurança do paciente. Hist. Enferm. Rev. Eletronica, 6(2), 299- 309,

Olímpio, M. A. C., Sousa, V. E. C., \& Ponte, M. A. V. (2016). O uso do bisturi elétrico e cuidados relacionados: revisão integrativa. Revista SOBECC, 21(3), 154-161. doi: 10.5327/Z1414-4425201600030006

Oliveira, J. L. C., Silva, S. V., Santos, P. R., Matsuda, L. M., Tonini, N. S., \& Nicola, A. L. (2017). Segurança do paciente: conhecimento entre residentes multiprofissionais. Einstein, 15(1), 50-7.

O'Riley, M. (2010). Electrosurgery in perioperative practice. J Perioper Pract. 20(9), 329-33. 10.1177/175045891002000903.

Panzetti, T. M. N., Silva, J. M. L. da, Dias, W. B., Costa, B. N. T., Bezerra, A. L. L., Borges, G. O., Silva, R. R., Ribeiro, L. M. S., Rocha, A. de C. M., Cardoso, D. L., Cagni, H. B. S., Vale, F. M., Costa, M. E. F., \& Silva, E. F. S. (2020). Nursing in the management of patient care in the perioperative period: an experience report. Research, Society and Development, 9(9), e124997082. doi: https://doi.org/10.33448/rsd-v9i9.7082 
Parra, R. L. C., Giannasttasio, M. B., \& Diniz, T. R. Z. (2012). O conhecimento dos circulantes de sala sobre a utilização do Bisturi Elétrico. Revista SOBECC, 17(4):24-32. Recuperado em 10 dezembro, 2020, de http://conic-semesp.org.br/anais/files/2019/trabalho-1000004980.pdf

Pereira, A. S., Shitsuka, D. M., Parreira, F. J., \& Shitsuka, R. (2018). Metodologia da pesquisa científica. Santa Maria: UFSM, NTE. Recuperado de https://repositorio.ufsm.br/bitstream/handle/1/15824/Lic_Computacao_Metodologia-Pesquisa-Cientifica.pdf?sequence=1

Piccoli, M., Berté, S. G., Carvalho, A. R. S., Alves, D. C. I., Silva, G. G. (2005). Risco de lesão na utilização da unidade de eletrocirurgia no periodo transoperatório. Rhttps://www.ciperj.org/imagens/eletrocirurgia.pdf

Rapsang, A. M. Y. G., Bhattacharyya, P. (2014). Marcapassos e cardioversores desfibriladores implantáveis - considerações gerais e anestésicas. Revista Bras. Anestesiol, 64(3), 205-214. doi: https://doi.org/10.1016/j.bjane.2013.02.005

Reis, C. T., Martins, M., Laguardia, J. (2013). A segurança do paciente como dimensão da qualidade do cuidado de saúde: um olhar sobre a literatura. Ciênc. saúde coletiva, 18(7), 2029-2036. http://dx.doi.org/10.1590/S1413-81232013000700018

Sandes, S. M. S., Costa, M. F., Santos, G. V., Freitas, L. P., Vasconcelos, A. C. P., \& Silva, L. S. L. (2019). Lesões provenientes de procedimento Cirúrgico: fatores relacionados. Revista SOBECC, 24(3): 161-167

Silva, Y. D. P. (2017). Enfermagem e a prevenção de queimaduras provocadas por eletrocautério monopolar. http://repositorio.faema.edu.br/bitstream/123456789/2093/1/TCC\%20Yslan.pd

Soares, C. R., Sousa, L., \& Castro, T. P. (2016). O papel do enfermeiro na humanização do paciente no período pré-operatório. https://www.even3.com.br/anais/mpct2017/46697-papel-do-enfermeiro-na-humanizacaoem-centro---cirurgico--uma-revisao-de-literatura/

Sociedade Brasileira de Enfermeiros de Centro Cirúrgico, Recuperação Anestésica e Centro de Material e Esterilização (SOBECC). (2009). Centro Cirúrgico, Recuperação Anestésica, Centro de Material e Esterilização: Práticas Recomendadas SOBECC. (5a ed.).

Souza, T. de C., Monteiro, D. da R., \& Tanaka, R. Y. (2020). Nursing care related to patient safety in hospitals: an integrative review. Research, Society and Development, 9(10), e8909109224. https://doi.org/10.33448/rsd-v9i10.9224

Spruce, L., \& Braswell, M. L. (2012). Implementing AORN recommended practices for electrosurgery. AORN J, 95(3), 373-84. 10.1016/j.aorn.2011.12.018.

Stuchi, D. de P. A., Cheble, C., \& Matayoshi, S. (2020). Fogo em campo cirúrgico - Medidas preventivas para cirurgia palpebral segura. Revista Bras. Oftalmol, 79(1), 56-58. http://dx.doi.org/10.5935/0034-7280.20200011.

Tomasi, D. L. I. (2013). Eletrocirurgia virtual baseada em física. Graduação em Ciência da Computação da UFRGS. Porto Alegre.

Tramontini, C. C., Galvão, C. M, Claudio, C. V., Ribeiro, R. P. \& Martins, J. T. (2016). Composition of the electrocautery smoke: integrative literature review. Revista Esc Enferm USP, 50(1), 144-53. http://dx.doi.org/10.1590/S0080-623420160000100019

Tufanaru, C., Munn, Z., Aromataris, E., Campbell, J. \& Hopp, L. (2017). Systematic reviews of effectiveness. In Aromataris E., Munn, Z. (Eds.), Joanna Briggs Institute Reviewer's Manual. Adelaide:The Joanna Briggs Institute.

Ulmer, B. C. (2002). Use of electrosurgery in the perioperative setting. Plast Surg Nurs, 22(4), 173-8. http://dx.doi.org/10.1097/00006527-20022204000003.

Ursi, E. S. (2005). Prevenção de lesões de pele no perioperatório: revisão integrativa da literatura. https://www.scielo.br/pdf/rlae/v14n1/v14n1a17.pdf.

Wegner, W., Silva, S. C., Kantorski, K. J. C., Predebon, C. M., Sanches, M. O., \& Pedro, E. N. R. (2016). Educação para cultura da segurança do paciente: Implicações para a formação profissional. Escola Anna Nery, 20(3). http://dx.doi.org/10.5935/1414-8145.20160068 
Research, Society and Development, v. 10, n. 1, e1210111203, 2021 (CC BY 4.0) | ISSN 2525-3409 | DOI: http://dx.doi.org/10.33448/rsd-v10i1.11203

Wicker, P., \& Plowes, D. (2000). Eletrocirurgia em Prática Perioperatória. Jornal Britânico de Enfermagem Perioperatória, 10 (4), 221-226. 10.1177 / 175045890001000408

Xavier, L. S. G. (2017). Segurança elétrica na cirurgia. http://extranet.hgf.ce.gov.br/jspui/bitstream/123456789/186/1/TCR_ 2017_Anestesiologia_xavier_luisadesousagomes.pdf 